\title{
Patterns of failure after the reduced volume approach for elective nodal irradiation in nasopharyngeal carcinoma
}

\author{
Ki Ho Seol, MD, Jeong Eun Lee, MD \\ Department of Radiation Oncology, Kyungpook National University School of Medicine, Daegu, Korea
}

\begin{abstract}
Purpose: To evaluate the patterns of nodal failure after radiotherapy (RT) with the reduced volume approach for elective neck nodal irradiation (ENI) in nasopharyngeal carcinoma (NPC).

Materials and Methods: Fifty-six NPC patients who underwent definitive chemoradiotherapy with the reduced volume approach for ENI were reviewed. The ENI included retropharyngeal and level II lymph nodes, and only encompassed the echelon inferior to the involved level to eliminate the entire neck irradiation. Patients received either moderate hypofractionated intensitymodulated RT for a total of $72.6 \mathrm{~Gy}$ (49.5 Gy to elective nodal areas) or a conventional fractionated three-dimensional conformal RT for a total of 68.4-72 Gy (39.6-45 Gy to elective nodal areas). Patterns of failure, locoregional control, and survival were analyzed. Results: The median follow-up was 38 months (range, 3 to 80 months). The out-of-field nodal failure when omitting ENI was none. Three patients developed neck recurrences (one in-field recurrence in the $72.6 \mathrm{~Gy}$ irradiated nodal area and two in the elective irradiated region of 39.6 Gy). Overall disease failure at any site developed in 11 patients (19.6\%). Among these, there were six local failures (10.7\%), three regional failures $(5.4 \%)$, and five distant metastases $(8.9 \%)$. The 3 -year locoregional control rate was $87.1 \%$, and the distant failure-free rate was $90.4 \%$; disease-free survival and overall survival at 3 years was $80 \%$ and $86.8 \%$, respectively. Conclusion: No patient developed nodal failure in the omitted ENI site. Our investigation has demonstrated that the reduced volume approach for ENI appears to be a safe treatment approach in NPC.
\end{abstract}

Keywords: Nasopharyngeal carcinoma, Lymphatic Irradiation, Treatment failure

\section{Introduction}

Nasopharyngeal carcinoma (NPC) shows markedly geographic and racial variations in incidence. South Korea is known to show an intermediate incidence, which is lower than that of an endemic area and higher than that of the Western world. According to the most recent annual report of cancer statistics in South Korea, NPC accounted for $0.2 \%$ of all malignancies [1]. NPC has a tendency of early lymphatic spread and a higher incidence of cervical lymph node metastasis among head and neck cancers [2]. Because of the risk of lymph node metastasis, the fields of radiotherapy (RT) for NPC traditionally encompass the primary disease and involved neck nodes, as well as the entire draining lymphatic regions to the lower neck.

Advances in imaging technology have substantially improved the ability to detect nodal metastases and knowledge about the patterns of lymphatic spread. The study by Tang et al. [3], which was based on magnetic resonance imaging (MRI), showed that only $0.3 \%$ of patients without the first station lymph node showed spread to level IV or supraclavicular lymph nodes,

Received 13 January 2016, Revised 21 February 2016, Accepted 15 March 2016.

Correspondence: Jeong Eun Lee, MD, Department of Radiation Oncology, Kyungpook National University School of Medicine, 680 Gukchaebosang-ro, Jung-gu, Daegu 41944, Korea. Tel: +82-53-420-5353, Fax: +82-53-426-3303, E-mail: jelee@knu.ac.kr

(c) This is an Open Access article distributed under the terms of the Creative Commons Attribution Non-Commercial License (http://creativecommons.org/ licenses/by-nc/4.0/) which permits unrestricted non-commercial use, distribution, and reproduction in any medium, provided the original work is properly cited.

www.e-roj.org 
skipping the second station. A meta-analysis of 2,920 patients from 13 studies using MRI as the main imaging modality by Ho et al. [4] showed that nodal metastases followed an orderly pattern and the probability of skip metastasis between levels varied between $0.5 \%$ and $7.9 \%$. According to these results, the rarity of skip metastasis in NPC lymphatic drainage provides a basis to eliminate irradiation of the entire cervical lymph nodes, and only encompass the echelon inferior to the involved level.

Furthermore, treatment of a large field to the neck is associated with substantial morbidities, both early and late. Acute toxicities include mucositis and dermatitis, especially if concurrent chemo-radiation is utilized; late toxicities may include xerostomia, neck fibrosis, lymphedema, cranial neuropathy, endocrine dysfunction, trismus, eyeball damage, and deafness [5]. If selective neck irradiation can be safely implemented in patients with limited nodal disease burden, selective neck irradiation may improve the tolerability of RT, as well as patient compliance and quality of life. The introduction of intensity modulated RT (IMRT) has a more favorable balance between target coverage and the sparing of adjacent organs at risk. In South Korea, the RT techniques for NPC have been advanced remarkably to adopt the IMRT technique [6].

Since 2006, our institution's approaches have been geared towards reducing the lower neck fields or selecting treatment of the neck lymph nodes during the treatment of NPC. The aim of this report is to study the clinical outcomes and patterns of failure after RT with individually tailored fields of elective neck nodal irradiation for NPC at a single institution.

\section{Materials and Methods}

\section{Patients and pretreatment evaluation}

All patient cases with histologically approved NPC who received definitive aimed RT between September 2006 and May 2013 at Kyungpook National University Hospital were retrospectively reviewed. Since September 2006, 70 NPC patients without evidence of distant metastasis were treated with a reduced volume approach for elective nodal irradiation (ENI). Fourteen patients were excluded due to incompletion of planned treatment, insufficient evaluation for response, follow-up lost and follow-up of fewer than 3 months. Fiftysix patients were eligible for this analysis. Stage and treatment information for each patient was then obtained.

All patients underwent a complete medical history interview and physical examination, nasopharyngoscopy, ultrasonographic evaluation of neck nodes, computed tomography (CT) of the head and neck, and positron emission tomography/computed tomography (PET/CT). In selected patients who were suspected of skull base involvement, we performed an MRI of the skull base.

Because the initial diagnosis and treatment for this study's patients began in September 2006, patients were initially staged according to the 2002 American Joint Committee on Cancer (AJCC) system (6th edition). After the publication of the 2010 AJCC system, all patients were restaged using the new system (7th edition). All patients with positive lymph nodes in the cervical region were distinguished according to the criteria described by van den Brekel et al. [7] (i.e., shortest axis of $\geq 11$ $\mathrm{mm}$ in the jugulodigastric regions, or $>10 \mathrm{~mm}$ in other cervical regions).

\section{Radiotherapy}

We treated patients with NPC by delivering either moderate hypofractionation scheduled IMRT or conventional fractionation scheduled three-dimensional conformal radiotherapy (3D-CRT). IMRT was delivered to 38 patients and 3D-CRT was delivered to 18 patients. The median overall treatment time of RT was 57 days (range, 44 to 71 days). All patients were immobilized in the supine position with a thermoplastic mask. After administration of intravenous contrast material, 3-mm CT slices were acquired from the vertex to the carina. All target volumes were delineated sliceby-slice on the treatment planning CT scan.

The gross target volume (GTV) was defined as the gross tumor and involved regional nodes, which were defined by CT and PET/CT scans in all patients. The clinical target volumes (CTV) were individually delineated on the basis of tumor invasion patterns. High-risk subclinical disease (CTV1) was defined as the GTV plus 1-5 mm margin for high-risk microscopic extensions including the entire nasopharynx, both retropharyngeal nodes (RPNs) and the level of involved nodes. The low-risk subclinical area (CTV-2) was defined by adding 2-5 $\mathrm{mm}$ margin to CTV-1 and the next neck level of CTV-1 areas based on tumor location and size, at the discretion of the radiation oncologist. Each CTV was expended to the planning target volume (PTV). The PTV1 was defined by adding a 3-5 $\mathrm{mm}$ margin to the GTV, dependent on the GTV proximity to critical structures (e.g., brainstem, spinal cord, parotid gland, or brachial plexus). A 3-5 mm margin was used for expansion to CTV-1 and CTV-2. PTV2 (CTV-1 + 3-5 mm margin) covered areas considered at high risk for potential microscopic disease. PTV3 (CTV-2 + 3-5 mm margin) included the clinically negative cervical lymphatic pathway. 
In IMRT planning, we used a simultaneously integrated boost IMRT technique. The moderate hypofractionation scheduled IMRT delivered 72.6 Gy at 2.2 Gy per fraction to PTV1, 66 Gy at 2.0 Gy per fraction to PTV2, and 49.5 Gy at 1.5 Gy per fraction to PTV3. The conventional fractionation scheduled 3D-CRT delivered a total dose of 68.4-72 Gy (median, 72 Gy) at 1.8-2.0 Gy per fraction to the PTV1 and PTV2 with a reduced dose (median, 39.6 Gy; range, 39.6 to $45 \mathrm{~Gy}$ ) to the PTV3 (elective neck lymphatics).

ENI always included the uninvolved levels II, III, and Va, but omitted level Ib, IV, and Vb nodes depending on involved lymph node location. The level la and level VI nodal areas were not irradiated in all patients. The level lb was not routinely included for ENI, unless an involved node in level lb was observed on imaging. In ENI for lower neck, the next station nodal area of the involved lymph node level was included in the volume for ENI, and not the more distal parts. More specially, the patient with enlarged level II lymph nodes was prophylactically irradiated to the level III nodal area, and was not irradiated to lymph node area of level IV, Vb, and supraclavicular area. In case of patients with only unilateral lymph node involvement (N1 disease), ENI of the ipsilateral neck included the next nodal station of the involved nodal area, and ENI of the uninvolved contralateral neck included only the level II, III, and Va nodal areas (Fig. 1).

\section{Chemotherapy}

Concurrent chemoradiotherapy (CCRT) using capecitabine and cisplatin (XP) or cisplatin alone for 2 to 3 cycles was used in all

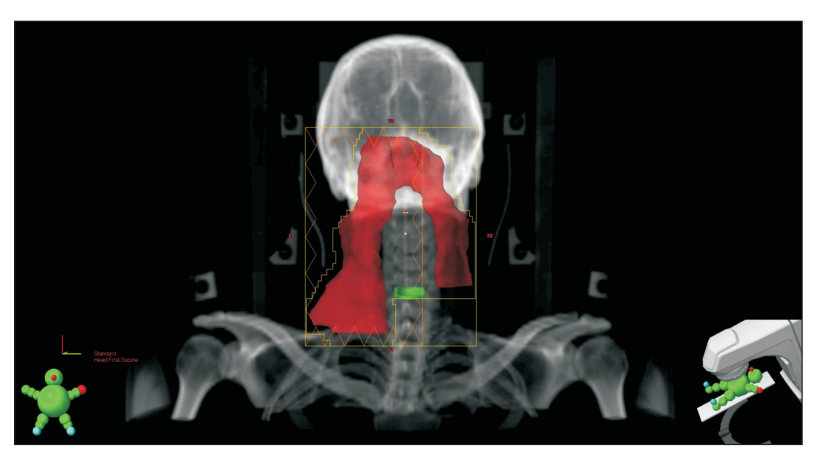

Fig. 1. An example of the tailored elective neck nodal irradiation (ENI) for a patient with cT3N1 nasopharyngeal carcinoma. The beam's-eye-view display with a digitally reconstructed radiograph shows the clinical target volume (red) including an electively irradiated nodal area for a dose of 4,950 cGy. The green shows the cricoid cartilage. On the side without nodal involvement (except retropharyngeal node), ENI included only RPN, levels II, III, and Va. ENI did not routinely include the level I region. patients. In addition, 32 patients with advanced tumor disease or advanced neck nodal disease also received neoadjuvant or adjuvant chemotherapy. Chemotherapy was not administered uniformly to all patients according to any single protocol but was instead used at the discretion of the attending physician in individual cases.

\section{Follow-up}

Each patient was assessed at regular intervals for treatment response and toxicity both during and after RT. During RT, patients were evaluated once per week. At the end of treatment, patients were followed up 1 month after RT and every 3 months during the first and second years and every 6 months during the next 3 years, then once every year afterwards. Endoscopy and PET/CT were performed for all patients about 2-3 months after the completion of treatment. During the follow-up session, CT to the head and neck region, $\mathrm{PET} / \mathrm{CT}$, and a physical examination were performed. When locoregional recurrences were suspected in endoscopy, CT, MRI or $\mathrm{PET} / \mathrm{CT}$, biopsies were performed for confirmation.

\section{Definition of failure site}

For patients with locoregional failure, the image scans of recurrent tumors were transferred to the pretreatment planning CT. The exact site and extent of each tumor was then compared with the pretreatment planning CT data sets, focusing on the $95 \%$ isodose lines of the prescription dose. Failures were categorized according to the criteria described by Dawson et al. [8] as occurring inside or outside the previously irradiated targets, depending on the location of $\mathrm{V}_{\text {fail }}$ : 'in field' if $95 \%$ of $\bigvee_{\text {fail }}$ was within the $95 \%$ isodose, 'marginal' if $20 \%$ to $95 \%$ of $V_{\text {fail }}$ was within the $95 \%$ isodose, or 'outside' if less than $20 \%$ of $V_{\text {fail }}$ was inside the $95 \%$ isodose.

\section{Endpoints and statistical methods}

The follow-up period was measured from the date of completion of RT to the date of death, date of telephone survey or date of the last clinic visit before analysis. The primary endpoint was the relapse rate within the unirradiated nodal areas. Secondary endpoints included patterns of nodal relapse, locoregional control (LRC), distant failure-free (DFF), overall survival (OS), and disease-free survival (DFS). The rates of LRC, DFF, OS, and DFS were estimated using the KaplanMeier method. The differences were compared with the logrank test. All statistical tests were two-sided, and p-values of $\leq 0.05$ were considered significant. All statistical analysis was performed using SPSS ver. 19.0 (IBM, Armonk, NY, USA). 


\section{Results}

Forty-two male and 14 female patients were included in this analysis. Median age was 51.5 years old, with a range between 21 and 75 years. Pathologic findings included keratinizing squamous cell carcinoma in 44 patients, nonkeratinizing carcinoma (differentiated subtypes) in two patients and nonkeratinizing carcinoma (undifferentiated subtype) in 10 patients. Patient characteristics are listed in Table 1.

The distribution of $\mathrm{T}$ stages and $\mathrm{N}$ stages was as follows: 23 T1 patients; eight T2 patients; 14 T3 patients; 11 T4 patients; 13 No patients; 18 N1 patients; 19 N2 patients; and six N3 patients. There were seven (12.5\%) patients with stage I; 14 (25.0\%) patients with stage II; 19 (33.9\%) patients with stage

Table 1. Patient and tumor characteristics ( $n=56)$

\begin{tabular}{|c|c|}
\hline Characteristic & No. of patients $(\%)$ \\
\hline \multicolumn{2}{|l|}{ Sex } \\
\hline Male & $42(75.0)$ \\
\hline Female & $14(25.0)$ \\
\hline Age (yr), median (range) & $51.5(21-75)$ \\
\hline \multicolumn{2}{|l|}{ Histologic type } \\
\hline Keratinizing squamous cell carcinoma & $44(78.6)$ \\
\hline \multicolumn{2}{|l|}{ Nonkeratinizing carcinoma } \\
\hline Dedifferentiated subtype & $2(3.6)$ \\
\hline Undifferentiated subtype & $10(17.8)$ \\
\hline \multicolumn{2}{|l|}{ T stage } \\
\hline $\mathrm{T} 1$ & $23(41.1)$ \\
\hline T2 & $8(14.3)$ \\
\hline T3 & $14(25.0)$ \\
\hline T4 & $11(19.6)$ \\
\hline \multicolumn{2}{|l|}{ N stage } \\
\hline No & $13(23.2)$ \\
\hline N1 & $18(32.1)$ \\
\hline N2 & $19(33.9)$ \\
\hline N3 & $6(10.7)$ \\
\hline \multicolumn{2}{|l|}{ Overall stage } \\
\hline Stage I & $7(12.5)$ \\
\hline Stage II & $14(25.0)$ \\
\hline Stage III & $19(33.9)$ \\
\hline Stage IV & $16(28.6)$ \\
\hline \multicolumn{2}{|l|}{ Chemotherapy } \\
\hline CCRT alone & $24(42.9)$ \\
\hline CCRT + adjuvant & $18(32.1)$ \\
\hline Neoadjuvant + CCRT & $13(23.2)$ \\
\hline Neoadjuvant + CCRT + adjuvant & $1(1.8)$ \\
\hline \multicolumn{2}{|l|}{ Radiotherapy method } \\
\hline IMRT & 38 (67.9) \\
\hline 3D-CRT & 18 (32.1) \\
\hline
\end{tabular}

CCRT, concurrent chemoradiotherapy; IMRT, intensity modulated radiotherapy; $3 \mathrm{D}-\mathrm{CRT}$, three-dimensional conformal radiotherapy.
III; and 16 (28.6\%) patients with stage IV (Table 1). Regarding the use of chemotherapy, 24 patients (42.9\%) received CCRT alone, 13 patients (23.2\%) received neoadjuvant chemotherapy with CCRT, and 18 patients (32.1\%) received CCRT with adjuvant chemotherapy. The other one patient (1.8\%) received neoadjuvant chemotherapy, CCRT, and adjuvant chemotherapy.

The median follow-up time was 38 months (range, 3 to 80 months). After completion of treatment, all patients achieved a complete remission.

Overall disease failure at any site developed in 11 patients (19.6\%). Among these patients there were six local failures (10.7\%), three regional failures (5.4\%), and five distant metastases (8.9\%) (Fig. 2).

Six patients developed local recurrence: four patients failed in the nasopharynx, one patient experienced both local recurrence in the nasopharynx and in-field regional recurrence in the level II neck area, and one patient experienced a local failure described as marginal recurrence adjacent to the nasopharynx. At the time of this analysis, there was no outof-field neck node failure in unirradiated neck levels. Table 2 summarizes the failure patterns of nodal recurrence.

Three patients had neck node failures. One patient (female/39 years old) had T1N2 disease with bilateral nodal disease at level II at the time of disease presentation. She was treated with CCRT (72.6 Gy of IMRT and 3 cycles XP). However, she complained of a left neck mass at 20 months after primary treatment, and fine-needle aspiration confirmed the presence of nodal recurrence. She was undergoing salvage neck node dissection and still survived without evidence of disease for 31 months. Another patient (male/59 years old) had T1N1 disease with multiple involvements of right neck level II-V nodal areas. He was treated with CCRT (72 Gy of 3D-CRT and 2 cycles XP). However, he had regional recurrence in contralateral neck nodes at 12 months after primary treatment and subsequently distant metastases were detected in the lung and the liver 15 months after primary treatment. He underwent salvage

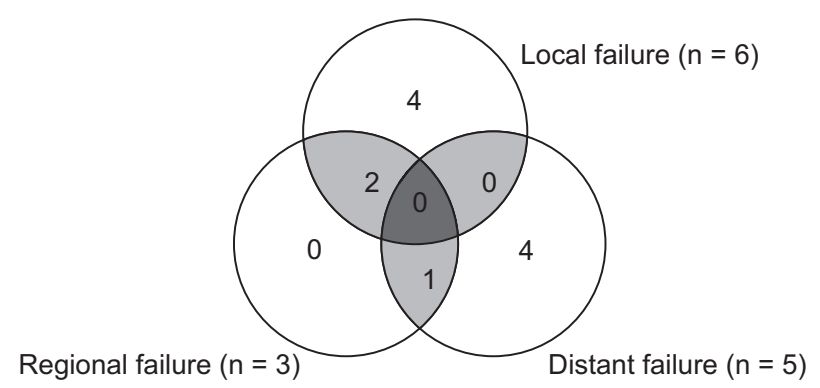

Fig. 2. Patterns of failure. 
Table 2. Patterns of nodal failure $(n=3)$

\begin{tabular}{|c|c|c|c|c|c|c|c|c|}
\hline Patient & $\begin{array}{l}\text { Sex/age } \\
(y r)\end{array}$ & $\begin{array}{l}\text { Initial } \\
\text { stage }\end{array}$ & $\begin{array}{c}\text { Region of relapse } \\
\text { neck node }\end{array}$ & $\begin{array}{l}\text { Irradiated dose of } \\
\text { relapsed region (Gy) }\end{array}$ & Failure pattern & $\begin{array}{l}\text { Time of neck node } \\
\text { recurrence (mo) }\end{array}$ & $\begin{array}{c}\text { Duration of } \\
\text { follow-up (mo) }\end{array}$ & $\begin{array}{l}\text { Survival } \\
\text { state }\end{array}$ \\
\hline 1 & $F / 39$ & T1N2 & Left II & 72.6 & In-field & 20 & 31 & Alive \\
\hline 2 & $\mathrm{M} / 59$ & T1N1 & Left II, III, Va & 39.6 & In-field (ENI site) & 12 & 22 & Death \\
\hline 3 & $M / 68$ & T2No & Right II, III & 39.6 & In-field (ENI site) & 17 & 31 & Death \\
\hline
\end{tabular}

ENI, elective neck nodal irradiation.

A

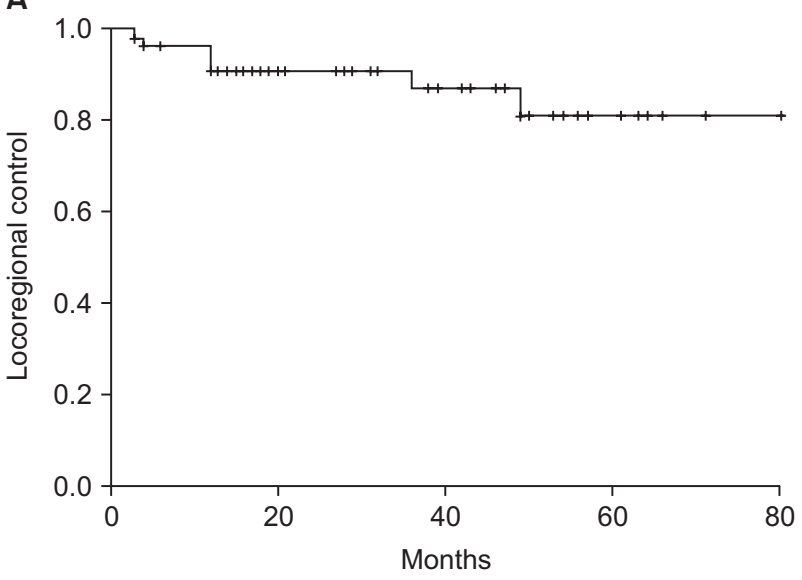

C

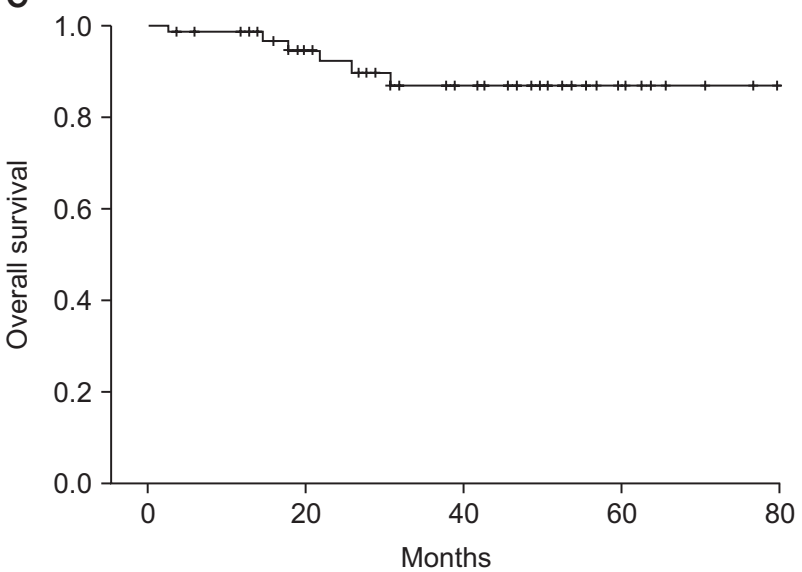

B

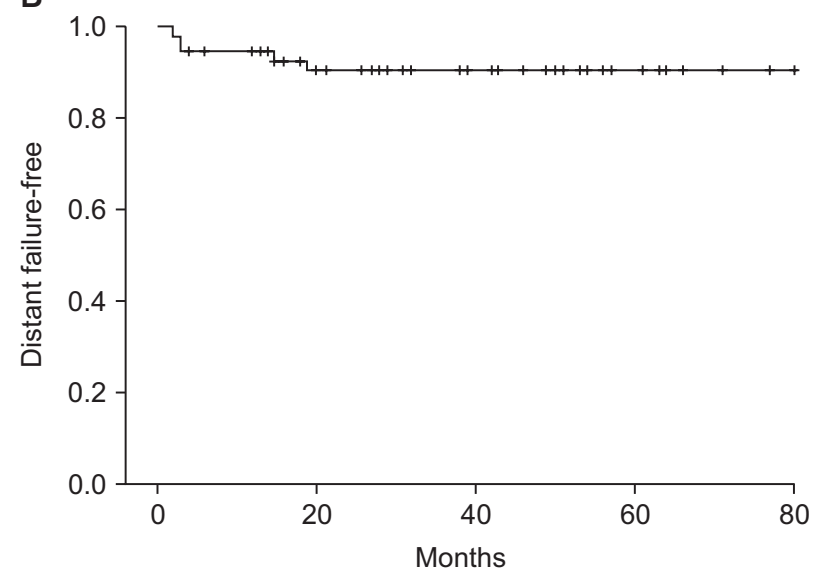

D

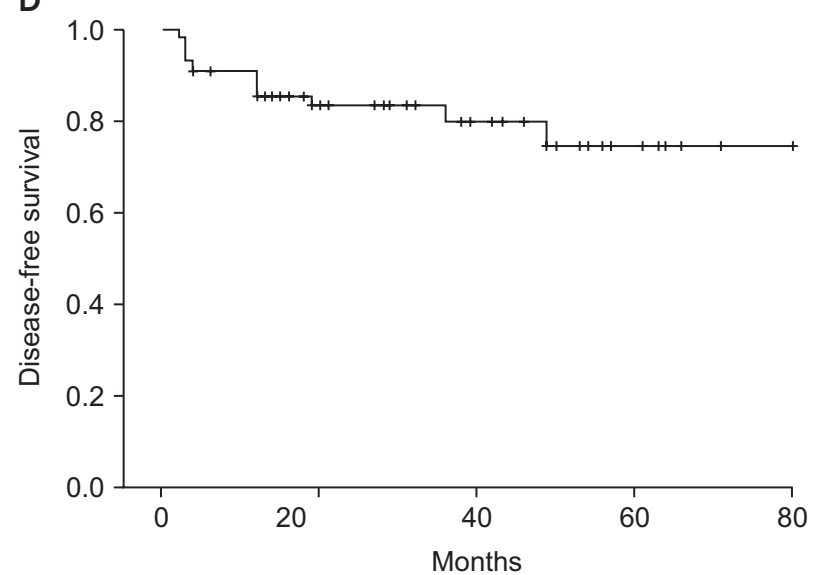

Fig. 3. Kaplan-Meier curves of (A) locoregional control, (B) 3-year distant failure-free, (C) 3-year overall survival, and (D) 3-year diseasefree survival rates.

surgery and postoperative RT. Despite of the treatment, he died because of lung metastases occurring 22 months after primary treatment. Similarly, the third patient (male/68 years old) who had T2NO disease with possible oropharynx extension had in-filed recurrence in the nasopharynx at 12 months after primary treatment (72 Gy of 3D-CRT and 2 cycles XP) and both neck failures in the electively irradiated area at 17 months after primary treatment. He received reirradiation on the recurrent neck region. However, several months later, multiple neck nodes, including intraparotid nodes, developed. Despite chemotherapy, he died due to disease progression 31 months after primary treatment.

Fig. 3 shows the clinical outcomes for all 56 patients. The 3-year LRC rate was 87.1\%, and DFF rate was 90.4\%; DFS and OS rates at 3 years were $80 \%$ and $86.8 \%$, respectively. In subgroup analysis, there were no significant differences in 3-year actuarial rates of $L R C$, DFF, and OS according to sex, age, performance state, pathology, AJCC stage, fractionation size, overall radiation duration, and treatment method. No other potential prognostic factor was found to be predictive of outcome. 


\section{Discussion and Conclusion}

There is an extensive submucosal lymphatic capillary plexus in the nasopharynx. Like most other head and neck primary cancers, lymphatic drainage of the nasopharynx is predominantly to the cervical lymph nodes. NPC has a higher incidence of cervical lymph node metastases compared with other head and neck cancers [2]. Because of the risk of lymph node metastasis, irradiation to the entire cervical lymph nodal drainage regions has been considered necessary. In a retrospective study reported by Lee et al. [9], 57 (30\%) of 189 patients who did not receive elective neck irradiation subsequently developed cervical lymph node recurrence. However, none of the seven regionally treated patients relapsed. Considering the results from this study, radiation oncologists treated all neck lymph node levels comprehensively with definitive-intent radiation therapy or prophylactic irradiation. Even in the current era of IMRT, protocols by the Radiation Therapy Oncology Group (RTOG) still adopt comprehensive coverage of bilateral levels I to $\mathrm{V}$ lymph node regions in CTV sparing level I only for NO patients in the RTOG 0225 and 0615 trials $[10,11]$. However, irradiation of a large field to the neck is associated with severe complications. Early toxicities include radiation dermatitis and mucositis, especially if concurrent chemo-radiation is utilized. Late toxicities may include xerostomia, neck fibrosis, lymphedema, cranial neuropathy, and endocrine dysfunction [5]. If the reduced volume of neck irradiation can be safely implemented in patients with limited nodal disease burden, reduced volume of neck irradiation may improve the tolerability of RT, leads to greater patient compliance and quality of life. Advances in RT, including image guidance and IMRT, have also allowed oncologists to be highly selective and accurate in treatment delivery. In the IMRT era, it is often up to the clinical judgment of the radiation oncologist to decide how much of the neck irradiated volume and dose [4].

$\mathrm{CT}, \mathrm{MRI}$, and PET/CT imaging carry important roles in the diagnosis and staging of NPC and have improved the ability to detect nodal metastases and the knowledge about the patterns of lymphatic spread [12-14]. These imaging techniques help to assess the patterns of metastatic nodal spreads in the neck in NPC and to improve disease control due to better delineation of initial disease extent, better irradiation planning, and better treatment delivery.

Several investigators have reported that NPC cervical metastasis follows an orderly spreading pattern inferiorly along the neck, and skip metastases in the neck region are relatively rare $[3,4,15,16]$. King et al. $[15]$ retrospectively reviewed the
MRIs of 150 patients with newly diagnosed NPC and reported that direct lymphatic spread from the primary tumor to the neck nodes without involvement of retropharyngeal nodes was rare, occurring in only $6 \%$ of patients with neck node metastases in the upper neck. The study by Tang et al. [3], which was based on MRIs of 924 patients, showed that only two patients (0.3\%) without retropharyngeal node or level II lymph node involvement had evidence of other lymph node involvement and lymph node metastases progress in an orderly fashion from higher level lymph nodes to lower level lymph nodes and rarely skip a site. They supposed that selective treatment of the neck lymph nodes for patients with NPC is feasible and prophylactic irradiation of level IV and supraclavicular fossa lymph nodes would not be necessary for patients who are negative for lymph node metastases. A meta-analysis of 2,920 patients from 13 studies using MRI as the main imaging modality by Ho et al. [4] showed that $85 \%$ of NPC patients had nodal involvement at the time of diagnosis. The most commonly involved nodal regions include retropharyngeal and level II lymph nodes. Nodal metastases followed an orderly pattern and the probability of skip metastasis between levels varied between $0.5 \%$ and $7.9 \%$. According to these results, limiting coverage to the selective nodal regions and eliminating the irradiation to the entire neck could be considered.

As such, the necessity of large treatment fields for whole neck irradiation has been called into question. Review of different IMRT series showed marked variation in the coverage of level I and the lower neck. The individually tailored approach is increasingly advocated for select NPC patients. Gao et al. [17] conducted a retrospective study of 410 NPC patients staged as node negative by computed tomography. With a median follow-up of 54 months, they reported that only one patient $(0.2 \%)$ experienced relapse outside the irradiation fields (level IV). Additionally, the reported 5-year OS rate, relapse-free survival rate, and DFS rate were $84.2 \%, 88.6 \%$, $80.1 \%$, respectively. They concluded that ENI by conventional technique to 50 to $56 \mathrm{~Gy}$ confined to RPN and levels II, III, and Va was safe for NO NPC. The retrospective study by Zeng et al. [18] showed that the 5-year OS, nodal recurrence-free survival, and distant metastasis-free survival rates of the upper neck group and the whole neck group were 93.6\% vs. 90.9\% ( $p=$ $0.553), 99.4 \%$ vs. $99.0 \%(p=0.278)$, and $98.8 \%$ vs. $94.9 \%$ ( $p$ $=0.128$ ), respectively in 270 patients with NO NPC who had undergone IMRT. A total of 3 neck recurrences were found, including 2 from the whole neck group and 1 from the upper neck group. The latter (0.6\%) was an out-of-field recurrence. 
They also concluded that prophylactic irradiation to the upper neck (levels II, III, and VA) may be feasible for patients with neck lymph node-negative NPC. Li et al. [19] reported the clinical outcomes of 301 patients with node-negative NPC. This randomized clinical trial showed that the 3-year OS, relapse-free survival, and metastasis-free survival rates were not different between upper vs. whole-neck prophylactic irradiation in node-negative NPC patients. They insisted that prophylactic upper neck irradiation is sufficient for patients with node-negative NPC. Chen et al. [20] reported the results of a prospective phase 2 study examining the effects of a tailored ENI approach in patients with N0-1 NPC. This study included not only node-negative patients but also patients with $\mathrm{N} 1$ disease as staged by CT who were treated with IMRT but did not receive ENI to level IV and Vb nodes. Seven out of 212 patients ( 4 in-field and 3 out-of-field) developed nodal failure. The 5 -year regional control rates of the entire group, NO patients, and N1 patients, were 95.6\%, 98.2\%, and 91.3\%, respectively. They concluded that the rate of out-of-field nodal failure when omitting ENI to levels IV and $\mathrm{Vb}$ in the clinically negative necks of patients with N0-1 NPC was extremely low.

In addition to NO and N1 patients, our study also included patients with N2 and N3 (involvement of upper neck nodal area) staged by PET/CT. The ENI included retropharyngeal and level II lymph nodes in all patients, and only encompassed the echelon inferior to the involved level to eliminate the entire neck irradiation. No nodal failures in the omitted ENI sites were observed in this study. Three patients (5\%) developed nodal failure: two nodal failures (3.6\%) were infield recurrence occurred in ENI site, and one patient (1.8\%) developed lymph node recurrence in level II that was inside of the radiation field delivering dose of 72.6 Gy. In our study, the causes of failure were, in order of frequency, local recurrence, distant metastasis, and regional recurrence (Fig. 2). Regional recurrence alone was none. Therefore, the individually tailored ENI was shown to be safe and feasible.

Omission of certain neck nodal areas (e.g., levels I, IV, and $\mathrm{Vb}$ ) can reduce the RT dose to surrounding normal tissues, especially the thyroid gland in the low neck. It is always advisable to spare as much normal surrounding tissue as possible. The main advantage is the possibility of reducing xerostomia by omitting level I. Despite evidence of almost full recovery of parotid salivary flow after IMRT, a study by Pow et al. [21] showed that oral health-related quality of life of NPC patients after IMRT was still significantly worse than at baseline because whole salivary flow had only recovered by $40 \%$; thus, dose reduction or sparing the submandibular and/or sublingual glands from radiation damage during NPC treatment is needed. Although our study lacked an analysis of complications and toxicity, we believe that the rates of certain late toxicities, including skin dystrophy, subcutaneous neck fibrosis, carotid occlusive disease, and xerostomia, would be low.

The NCCN guideline of head and neck cancers [22] recommends that elective irradiation to low risk and intermediate risk sites requires $44 \mathrm{~Gy}$ (2.0 Gy/fraction) to $63 \mathrm{~Gy}$ (1.6-1.8 Gy/fraction), depending on the estimated level of tumor burden. NPC have the better radiosensitivity when compared with the other squamous cell carcinomas of the head and neck [23]. And the combination of concurrent chemotherapy also enhance the radiation response $[24,25]$. In 3D-CRT of our study, the dose of median 39.6 Gy (range, 39.6 to 45 Gy; 1.8 Gy/fraction) was used for ENI of the low risk neck because of the spinal cord tolerance dose. Our study reported that two patients who were received a dose of 39.6 Gy experienced in-field recurrence occurred in PTV2 ENI site. In the era of IMRT, we used a higher dose of 49.5 Gy (EQD2 $44.2 \mathrm{~Gy}_{10}$ ) for ENI of low risk neck in order to improve the regional control rate.

The primary limitation of our study is the small sample size, which was restricted to include patients who received the individually tailored field of ENI instead of whole neck irradiation in a single institution. Because of the retrospective nature of the review, the results were subject to data collection and interpretation bias. Another limitation of this study was that the chemotherapy was not administered in a controlled manner but was instead used at the discretion of the attending physician. Clinical studies have confirmed that chemotherapy may eradicate subclinical disease and improve LRC, especially for locoregional advanced disease $[24,25]$. Future studies should address this issue.

In conclusion, no patient developed nodal failures in the omitted ENI site. Results from our study were comparable to those with whole neck irradiation reported by other centers. Our investigation demonstrates the reduced volume approach for ENI appears to be a safe treatment approach in NPC. We expect that minimizing the irradiated neck volume could potentially reduce the risk of toxicity and improve patient quality of life.

\section{Conflict of Interest}

No potential conflict of interest relevant to this article was reported. 


\section{Acknowledgments}

This research was supported by the Kyungpook National University Research Fund in 2012.

\section{References}

1. Korea Central Cancer Registry, National Cancer Center. Annual report of cancer statistics in Korea in 2012. Goyang: Ministry of Health and Welfare; 2014.

2. Sham JS, Choy D, Wei WI. Nasopharyngeal carcinoma: orderly neck node spread. Int J Radiat Oncol Biol Phys 1990;19:92933.

3. Tang $L$, Mao $Y$, Liu $L$, et al. The volume to be irradiated during selective neck irradiation in nasopharyngeal carcinoma: analysis of the spread patterns in lymph nodes by magnetic resonance imaging. Cancer 2009;115:680-8.

4. Ho FC, Tham IW, Earnest A, Lee KM, Lu JJ. Patterns of regional lymph node metastasis of nasopharyngeal carcinoma: a metaanalysis of clinical evidence. BMC Cancer 2012;12:98.

5. Lee AW, Ng WT, Hung WM, et al. Major late toxicities after conformal radiotherapy for nasopharyngeal carcinomapatient- and treatment-related risk factors. Int J Radiat Oncol Biol Phys 2009;73:1121-8.

6. Sung SY, Kang MK, Kay CS, et al. Patterns of care for patients with nasopharyngeal carcinoma (KROG 11-06) in South Korea. Radiat Oncol J 2015;33:188-97.

7. van den Brekel MW, Stel HV, Castelijns JA, et al. Cervical lymph node metastasis: assessment of radiologic criteria. Radiology 1990;177:379-84.

8. Dawson LA, Anzai Y, Marsh L, et al. Patterns of localregional recurrence following parotid-sparing conformal and segmental intensity-modulated radiotherapy for head and neck cancer. Int J Radiat Oncol Biol Phys 2000;46:1117-26.

9. Lee AW, Sham JS, Poon YF, Ho JH. Treatment of stage I nasopharyngeal carcinoma: analysis of the patterns of relapse and the results of withholding elective neck irradiation. Int J Radiat Oncol Biol Phys 1989;17:1183-90.

10. Lee N, Harris J, Garden AS, et al. Intensity-modulated radiation therapy with or without chemotherapy for nasopharyngeal carcinoma: radiation therapy oncology group phase II trial 0225. J Clin Oncol 2009:27:3684-90.

11. Lee NY, Zhang $Q$, Pfister DG, et al. Addition of bevacizumab to standard chemoradiation for locoregionally advanced nasopharyngeal carcinoma (RTOG 0615): a phase 2 multiinstitutional trial. Lancet Oncol 2012;13:172-80.

12. Sham JS, Cheung YK, Choy D, Chan FL, Leong L. Computed tomography evaluation of neck node metastases from nasopharyngeal carcinoma. Int J Radiat Oncol Biol Phys 1993; 26:787-92.

13. Abdel Khalek Abdel Razek A, King A. MRI and CT of nasopharyngeal carcinoma. AJR Am J Roentgenol 2012;198:11-8.

14. King AD, Ma BB, Yau YY, et al. The impact of 18 F-FDG PET/CT on assessment of nasopharyngeal carcinoma at diagnosis. $\mathrm{Br} J$ Radiol 2008:81:291-8.

15. King $A D$, Ahuja $A T$, Leung $S F$, et al. Neck node metastases from nasopharyngeal carcinoma: MR imaging of patterns of disease. Head Neck 2000;22:275-81.

16. Wang $X$, Li L, Hu C, et al. Patterns of level II node metastasis in nasopharyngeal carcinoma. Radiother Oncol 2008;89:28-32.

17. Gao Y, Zhu G, Lu J, et al. Is elective irradiation to the lower neck necessary for No nasopharyngeal carcinoma? Int J Radiat Oncol Biol Phys 2010;77:1397-402.

18. Zeng $L$, Sun $X M$, Chen $C Y$, et al. Comparative study on prophylactic irradiation to the whole neck and to the upper neck for patients with neck lymph node-negative nasopharyngeal carcinoma. Head Neck 2014;36:687-93.

19. $L i J G$, Yuan $X$, Zhang $L L$, et al. A randomized clinical trial comparing prophylactic upper versus whole-neck irradiation in the treatment of patients with node-negative nasopharyngeal carcinoma. Cancer 2013;119:3170-6.

20. Chen JZ, Le QT, Han F, et al. Results of a phase 2 study examining the effects of omitting elective neck irradiation to nodal levels IV and Vb in patients with $\mathrm{N}(0-1)$ nasopharyngeal carcinoma. Int J Radiat Oncol Biol Phys 2013;85:929-34.

21. Pow EH, Kwong DL, Sham JS, Lee VH, Ng SC. Can intensitymodulated radiotherapy preserve oral health-related quality of life of nasopharyngeal carcinoma patients? Int J Radiat Oncol Biol Phys 2012;83:e213-21.

22. National Comprehensive Cancer Network. Head and neck cancers version v.1.2015 [Internet]. Fort Washington, PA: National Comprehensive Cancer Network; c2015 [cited 2016 Mar 17]. Available from: http://www.ncen.org/professionals/ physician_gls/pdf/head-and-neck.pdf.

23. Fletcher GH, Million RR. Malignant tumors of the nasopharynx. Am J Roentgenol Radium Ther Nucl Med 1965;93:44-55.

24. Al-Sarraf M, LeBlanc M, Giri PG, et al. Chemoradiotherapy versus radiotherapy in patients with advanced nasopharyngeal cancer: phase III randomized Intergroup study 0099. J Clin Oncol 1998;16:1310-7.

25. Wee J, Tan EH, Tai BC, et al. Randomized trial of radiotherapy versus concurrent chemoradiotherapy followed by adjuvant chemotherapy in patients with American Joint Committee on Cancer/International Union against cancer stage III and IV nasopharyngeal cancer of the endemic variety. J Clin Oncol 2005;23:6730-8. 